\title{
The Restoration Technique for Recognition of Asterias Amurensis
}

\author{
Kang Kim ${ }^{1}$, Seong-Yoon Shin ${ }^{2 *}$ and Yang-Won Rhee ${ }^{2}$ \\ ${ }^{1}$ Dept. Of Tourism Information, Kangwon Tourism College, Korea \\ ${ }^{2}$ Dept. Of Computer Information Eng., Kunsan National Univ., Korea \\ * Corresponding Author \\ kkang@kt.ac.kr, \{s3397220,ywrhee\}@kunsan.ac.kr
}

\begin{abstract}
Asterias amurensis, also referred to as the Northern Pacific seastar, is so indiscriminate a predator that it deserves to be called pirates of the sea. They usually eat fish and shellfish including abalones and clams. Due to the high fertility rate, Asterias amurensis is one of the main culprits in devastating fishing grounds. Starfish are anywhere in the East Sea and South Sea. In recent years, however, their number has sharply increased in the West Sea. This paper aims to recognize and extract deformed Asterias amurensis through the use of the concave and convex feature points, central moment, and short lines and long lines. Another purpose of this paper is to restore Asterias amurensis using the radial symmetry. Marked by the characteristics that include pentaradiality and the ability to vary in outward shapes according to circumstances, Asterias amurensis is hard to recognize. With this in mind, this paper propose a simple method for improve rates of recognition of starfish. It will not be long before the restoration method proposed in this paper is of great help in eradicating Asterias amurensis.
\end{abstract}

Keywords: Asterias Amurensis, Central Moment, Radial Symmetry, Restoration Method

\section{Introduction}

Asterias amurensis is an invertebrate belonging to the phylum Echinoderm and to the class Asteroidea that lives throughout the northwestern Pacific. It is so indiscriminate a predator that it deserves pirates of the sea. Not only that, it looks abhorrent because it is hideously big and has mottled blue spots on their yellowish body [1]. Starfish, which means "not die," are kinds of fish and shellfish that eat oysters, ark clams, and abalones. That's why starfish are headaches for fishermen. It was 500 million years ago during the Ordovician Period that starfish first appeared on Earth [2]. Reportedly, 47 species of starfish live in Korea.

It is widely known that the increase in carbon dioxide emissions caused by accelerated industrialization is worsening global warming. The climate change is the main culprit for rising sea levels, local torrential rains, and heavy snowfalls. In addition to it, changes being made to land and marine ecosystems are expected to affect human health directly and indirectly. Due to global warming, gradual changes are occurring in the natural ecosystem, including plants' early flowering, birds' early spawning, habit loss among insects, plants and animals, white efflorescence on coastal areas, and reduced biodiversity [3].

Generally, starfish are categorized into two species: Asterias amurensis and Asterina pectinifera. While the latter's prey is chiefly dead fish, the former eats live fish and shellfish. It is no wonder that starfish are and will be tremendously damaging [4].

Starfish have no natural enemy in the marine ecosystem and have a high fertility rate. They are not life-threatening, except for the fatal wounds they inflict. It is effective, therefore, to 
capture starfish in the spawning season to eliminating starfish populations [5].

Currently, studies are vigorously in progress about how to deal with and utilize captured starfish. Research needs to be conducted on the image processing technique, which is used to capture Asterias amurensis and Asterina pectinifera causing much damage to fishing grounds [6-8].

There is a limit as to how starfish have to be captured, given that starfish rapidly propagate in number and size. That impels us to facilitate the use of underwater robots to capture starfish. Regarding how to capture starfish, an image processing technique should be in place to recognize starfish among input images [9].

Recent studies being conducted in Korea on the recognition of starfish are as follows. An improved Asterias amurensis recognition method based on the morphological characteristics of Asterias amurensis was proposed in [10]. They acquired better images to analyze recognition rates of starfish, and were also able to obtain much more satisfactory recognition rates for Asterias amurensis by adopting the most suitable adaptive filter method [11]. This technique [12] classifies the concave and convex features by using the multi-directional linear scanning, forms the candidate groups of the concave and convex feature points, decides the feature points of the candidate groups, and applies a convex hull algorithm to the extracted feature points.

Recently, human activities have increasingly expanded - in particular, in terms of international trade. As a result, the spread of introduced marine pests (IMPs) are considerably affecting the ecosystem and human economic activities. IMPs are introduced primarily through ballast water carried in ships' ballast tanks, through extraneous matter stuck on a hull, or when cultured fish are in transit. Such IMPs, once introduced, impact the ecosystem by destroying the habitats of indigenous animals and plants, inducing mutations in marine life, and reducing fishery resources In addition, IMPs make it costly to do research into how they are introduced, and to restore the disrupted ecosystem. Furthermore, IMPs cause much financial damage to fishermen who have been suffering from reduced profits.

Asterias amurensis is cold-blooded and has different skin colors. They are hard to recognize because of the radial symmetry which varies depending on circumstances. In addition, multiple populations of starfish are much harder to recognize, compared with those of single or compound populations. This paper proposes a method for obtaining enhanced rates of recognition of starfish. In the case that several populations of Asterias amurensis are present, the proposed simple method is available for recognition and restoration of Asterias amurensis. Rough on the restoration of the Asterias amurensis can be found in [13]. In this paper, we specifically discussed in detail.

\section{The Structural Features of Asterias Amurensis}

\subsection{Basic Features}

Asterias amurensis is a carnivorous species introduced from Kamchatka and Hotkaido and widespread across Korea. That is why Asterias amurensis is notorious among fishermen. Their legs are thinner than those of indigenous Asterina pectinifera. They are 20 tall on average, but bigger ones grow to be $40 \mathrm{~cm}$ and more. The image of Asterias amurensis is shown in Figure 1. 


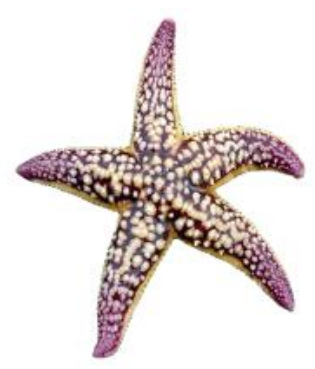

Figure 1. The Image of Asterias Amurensis

\subsection{The Concave and Convex Features}

Asterias amurensis has an arm that features pentaradial symmetry. The starfishspecific feature is used to detect the end of each arm as well as the inner boundary part where the arms meet each other. The structural features of Asterias amurensis are shown in Figure 2.

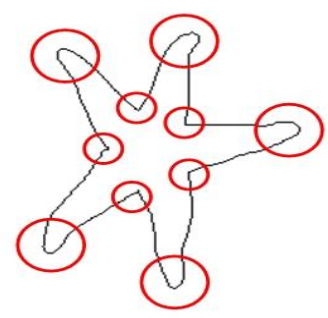

\section{Figure 2. The Structural Features of Asterias Amurensis}

Among the starfish candidate regions for the detected image boundaries from which noise is removed, any region where Pixel $_{\text {count }}$ - the number of pixels present in the $20 \mathrm{x} 20$ block - fails to meet the condition of the formula 1 is excluded from the candidate regions that have starfish features.

$$
\text { MIN }_{\text {count }}<\text { Pixel }_{\text {count }}<\text { MAX } X_{\text {count }}
$$

Starfish are marked by five arms with the concave and convex feature points each. Based on one concave point for each concave edge, with the adjacent points excluded, connecting the remaining two points leads to the formation of a star-shaped inner area; connecting each concave point from the center of the inner area allows the starfish to divide into five arms, each of which has pentaradial symmetry. Figure 3 shows the image of a starfish marked by pentaradial symmetry.

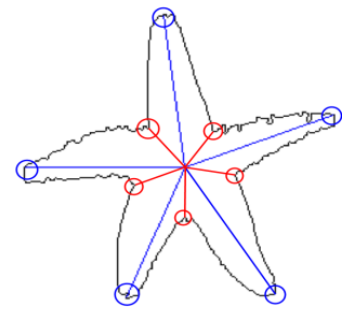

Figure 3. The Image of Pentaradial Symmetry 


\subsection{The Central Moment Feature}

Asterias amurensis has an arm that features pentaradial symmetry. The starfish- Based on one concave point for each concave edge, with the adjacent points excluded, connecting the remaining two points leads to the formation of a star-shaped inner area. The center of the inner area can be used as the central moment of the starfish. In starfish, the convex edges are so sensitive to movements that they are less likely than concave ones to be visible to other living organisms. As such, concave features are used more often than convex features. Figure 4 shows the image of the central moment of a radially symmetric starfish.

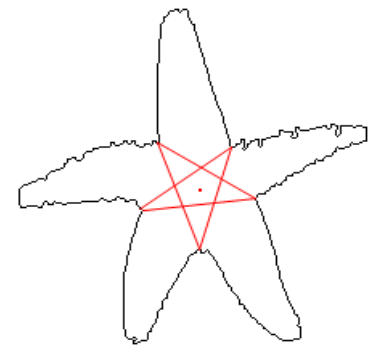

Figure 4. The Image of a Central Moment

\subsection{Short-Line and Long-Line Features}

Long-line refers to the line formed when each convex edge connects from the central moment, while short line to the line formed by connecting each concave endpoint from the central moment. Connecting a long line and a short line is equivalent to the equation of a straight line passing through the coordinates $A\left(x_{1}, y_{1}\right)$ and $B\left(x_{2}, y_{2}\right)$ for two pixels. The gradient of a straight line $m$ is $\tan \theta$. In other words, it is the difference between two sets of coordinate values, as shown in Equation 2. Equation 3 is equivalent to the equation of a straight line passing through the coordinates $A\left(x_{1}, y_{1}\right)$ and $B\left(x_{2}, y_{2}\right)$ for two pixels, whose gradient is $m$.

$$
\begin{aligned}
& m=\frac{\Delta y}{\Delta x}=\frac{y_{2}-y_{1}}{x_{2}-x_{1}} \\
& y=m\left(x-x_{1}\right)+y_{1}
\end{aligned}
$$

To ensure a connection between the two points, black pixels need to be found on the line where the gradient is identical. In this case, they have to be found in accordance with small variations through a comparison of the values of $x$ and $y$. Based on a function of $y=x$ where the gradient is 1 , if the gradient is lower than 1, the variations of the value of $x$ are smaller than those of the value of $y$. Then entering the $x$-value leads to obtaining the $y$-value, which is expressed in Equation 3. If the gradient is larger than 1, on the other hand, the variations of the value of $x$ are larger than those of the value of $y$. Then entering the $y$-value leads to obtaining the $x$-value, which is expressed in Equation 4.

$$
x=\frac{y+m x_{1}-y_{1}}{m}
$$

The variations for different gradients are shown in Figure. 5. 


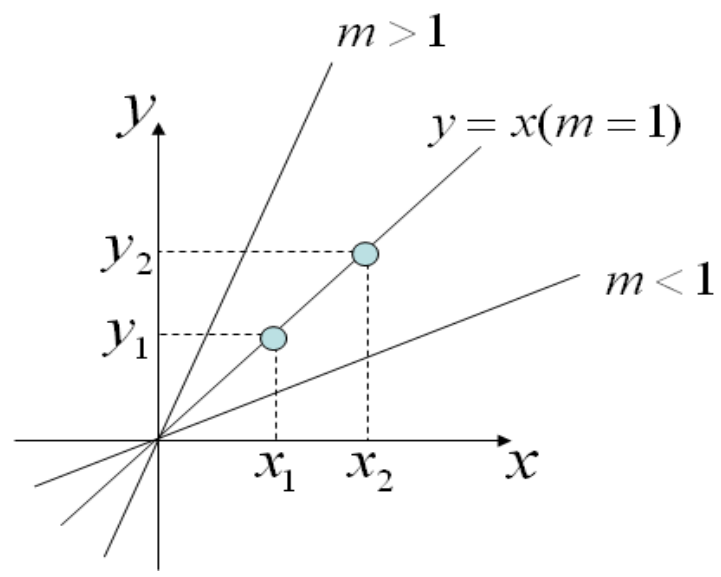

Figure 5. Variations by Gradient

The images of long and short lines are shown in Figure 6.

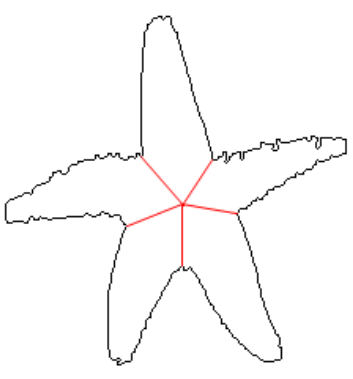

Short-Line Image

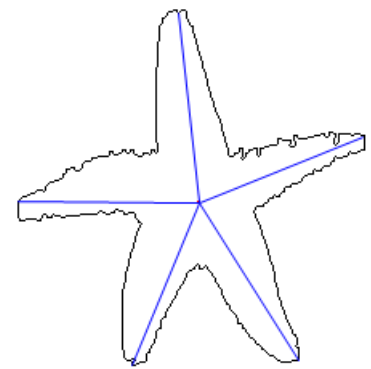

Long-Line Image

Figure 6. Short-line and Long-line Images

\section{Restoration of Asterias Amurensis}

For a damaged or deformed starfish population for which boundary correction is implemented, there can be difficulties in detecting edges of that region only. When restoring the region of the population using structural features, input images are preprocessed before boundary detection is conducted. Boundary correction is then used to remove noise, which is followed by boundary connection. What needs to be done next is finding the structural features, comparing the number of concave feature points, and identifying the type of the population. Concerning the restoration of the region of a starfish from the population by using the structural features, what is restored is a deformed population, not the original one. Any deformed population, which is different from the original population, refers to the one with the arms dismembered or the one whose dismembered arms are growing. It is such deformed populations that restoration is conducted for. Figure 7 shows the deformed population for which restoration was conducted. 


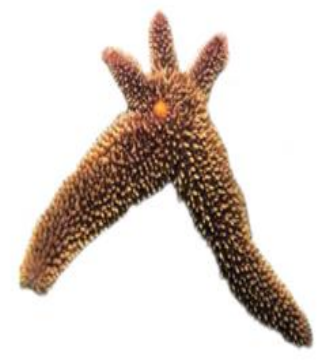

A Deformed Population

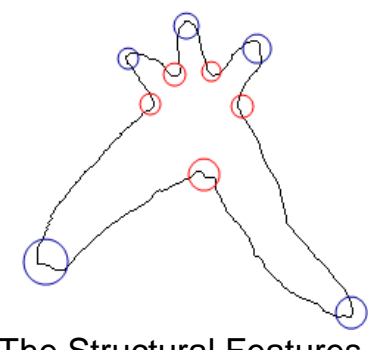

The Structural Features

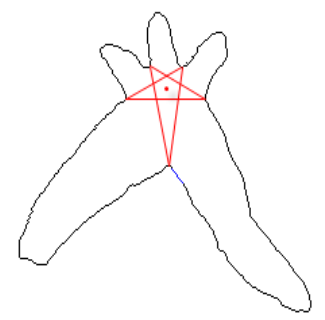

The Central Moment

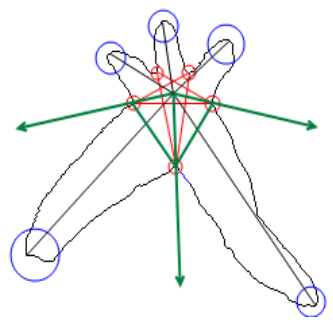

An Image of Radial Symmetry

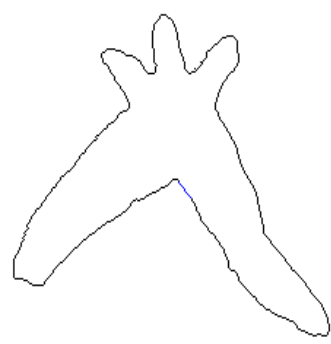

The Corrected Boundary

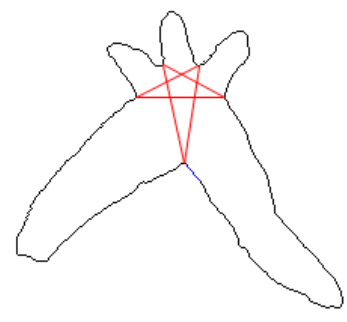

The Short-line Feature

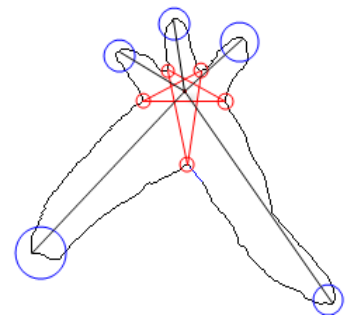

Long-line Connections

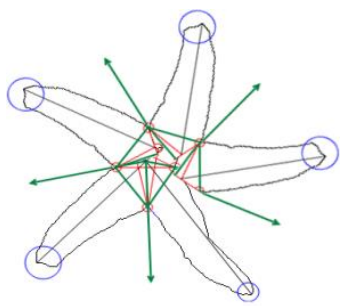

Starfish Restoration

Figure 7. Restoration of a Deformed Population 
The proposed method was used such that the structural features of a starfish as well as its pentaradial symmetry were used to restore the region of that starfish before restoring a single population. The number of long lines was the same as that of short lines, which allowed for morphological restoration. The result of region restoration is shown in Figure 8.

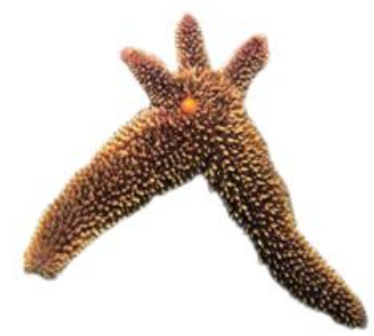

Original Image

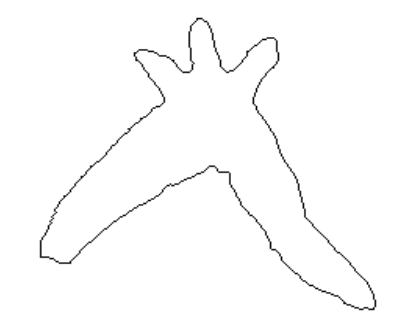

Detected Boundary Image

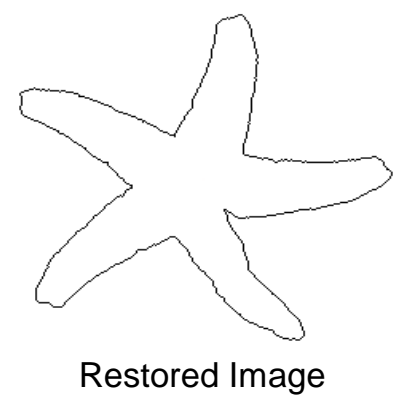

Figure 8. Region Restoration Using Structural Features and Radial Symmetry

The deformed population, before it was restored, had 21 long lines and short lines each. After restoration, it had 25 long lines and short lines, respectively. Restoration was, for the most part, conducted for deformed parts of it. Table 1 shows an analysis of the characteristics identified during the region restoration. 


\section{Table 1. Analysis of Characteristics during the Region Restoration Using the Structural Features and Radial Symmetry}

\begin{tabular}{|c|c|c|c|c|c|c|}
\hline \multicolumn{3}{|c|}{ Population } & Area & $\begin{array}{l}\text { Boundary } \\
\text { Length }\end{array}$ & $\begin{array}{l}\text { Number of } \\
\text { Long-Line }\end{array}$ & $\begin{array}{l}\text { Short-Line } \\
\text { Length }\end{array}$ \\
\hline \multirow{10}{*}{$\begin{array}{l}\text { Deformed } \\
\text { Population }\end{array}$} & \multirow{5}{*}{$\begin{array}{c}\text { Before } \\
\text { Restoration }\end{array}$} & 1 & 49065 & 730 & 5 & 5 \\
\hline & & 2 & 49506 & 867 & 5 & 5 \\
\hline & & 3 & 48106 & 821 & 4 & 4 \\
\hline & & 4 & 46578 & 769 & 4 & 4 \\
\hline & & 5 & 49912 & 756 & 3 & 3 \\
\hline & \multirow{5}{*}{$\begin{array}{c}\text { After } \\
\text { Restoration }\end{array}$} & 1 & 43182 & 1001 & 5 & 5 \\
\hline & & 2 & 47552 & 997 & 5 & 5 \\
\hline & & 3 & 49346 & 965 & 5 & 5 \\
\hline & & 4 & 46128 & 986 & 5 & 5 \\
\hline & & 5 & 45121 & 1012 & 5 & 5 \\
\hline
\end{tabular}

\section{Conclusion}

As the damage of IMPs become more severe over time, international organizations and many countries around the world are trying to come up with aggressive countermeasures. Major nations are investigating the actual condition of IMPs and seeking ways to curb or eliminate IMPs. This paper proposed a method for restoring deformed starfish populations. This method was designed to improve recognition rates for images of the starfish collected by underwater robots. The input images obtained by the underwater robots were for a single starfish population and based on optimal distances and directions. Starfish have significant structural features: concave and convex feature points. With this in mind, the proposed method was geared toward distinguishing the concave and convex features of a starfish. During the experiments in which the region of a starfish image was restored from a deformed population, the deformed population showed high restoration rates. When it comes to recognizing starfish in a deformed population, the application of the proposed method based on region restoration will be very effective in eradicating starfish.

\section{References}

[1] http://ko.wikipedia.org/wiki/\%EC\%95\%84\%EB\%AC\%B4\%EB\%A5\%B4\%EB\%B6\%88\%EA\%B0\%80\% EC\%82\%AC\%EB\%A6\%AC (2013).

[2] http://blog.naver.com/sjyoo53?Redirect=Log\&logNo=80054044656 (2013)

[3] KONETIC, "Influence of the Global Warming in the Korean Peninsula," Environmental Industry \& Technology Information, no. 20, (2008).

[4] Ji-Soo Jang, "Technology Trends for Starfish Utilization”, Korea Institute of Patent Information, (2003), pp. $1-10$.

[5] Se-Han Jang, "Consider of Role on Starfish in Farming Environment", Journal of College of Marine Science, Gyeongsang National University (2000).

[6] M. Hatanaka and M. Kosaka, "Biological studies on the population of the starfish, Asterias amurensis, in Sendai bay”, Tohoku J. Agric. Res., vol. 9, no. 3, (1958), pp. 159-178.

[7] M. S. Park and B. Y. Kim, "Feeding Behaviour of the Starfish, Asterias amuresis (Lutken)", Research Report of National Fisheries Research \& Development Agency, (1985). 
[8] Allan K. Fukuyamaa \& John S. Olivera, "Sea Star and Walrus Predation on Bivalves in Norton Sound, Bering Sea, Alaska", Ophelia, vol. 24, issue 1, (1985), pp. 17-36.

[9] Seung-Hee Kim, "The TPDR Area Edge And Structural Features Detection For Starfish Recognition", Degree of Doctor of Philosophy in Science, Graduate School of Kwan Dong University, (2008).

[10] Hyun-Deok Shin, Young-Cheol Jeon, "An Improved Asterias Amurensis Recognition Method Based on Morphological Characteristics Analysis Techniques," Journal of The Korea Society of Computer and Information, vol. 17, no. 10, (2012), pp.61-69.

[11] Jong Ik Kim, Hyun Bo Shim, Sung Rak Kim, "The Amur Starfishes Recognition Using the Adaptive Filter," Journal of Information and Communication Engineering, vol. 17, no. 4, (2013), pp. 922-934.

[12] Hyun-Deok Shin, Young-Cheol Jeon, "Feature Extraction of Asterias Amurensis by Using the MultiDirectional Linear Scanning and Convex Hull," Journal of The Korea Society of Computer and Information, vol. 16, no. 3, (2011), pp.99-107.

[13] Kang Kim , Seong-Yoon Shin, Yang-Won Rhee, "The Restoration Method for Recognition of Asterias Amurensis," Advanced Science and Technology Letters, Bioscience and Medical Research, vol.33 (2013), pp.1-4.

\section{Authors}

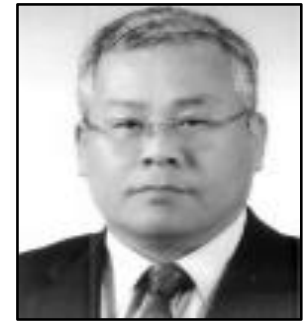

Kang Kim, He is a professor of Dept. Of Tourism Information, Kangwon Tourism College, Taebaek, 235-010, Korea. His research interests include tourism information, tourism enterprise development, and IT fusion expert training.

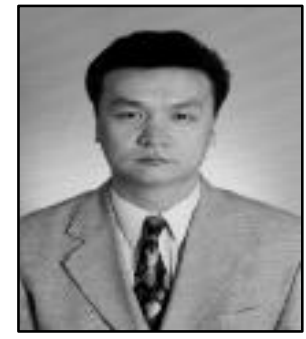

Seong-Yoon Shin, Corresponding Author, he is received his M.S. and Ph.D degrees from the Dept. of Computer Information Engineering of Kunsan National University, Kunsan, Korea, in 1997 and 2003, respectively. From 2006 to the present, he has been a professor in the same department. His research interests include image processing, computer vision, and virtual reality.

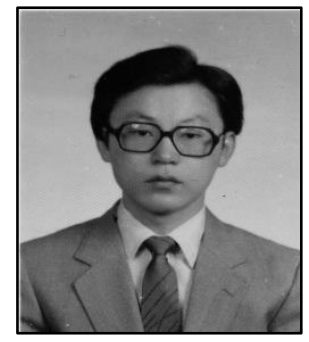

Yang-Won Rhee, he received his M.S. from the Dept. of Computer Science of Yonsei University, Ph. D. degree from the Dept. of Computer Science of Soongsil University. From 1986 to the present, he has been a professor in the Dept. of Computer Information Engineering, Kunsan National University, Korea. His research interests include image processing, computer vision, and virtual reality. 
International Journal of Bio-Science and Bio-Technology Vol.6, No.3 (2014) 